PREPARED FOR THE U.S. DEPARTMENT OF ENERGY, UNDER CONTRACT DE-AC02-76CH03073

PPPL-3962

PPPL-3962

UC-70

Stabilization of Sawtooth Oscillations

by the Circulating Energetic lons

by

Ya.I. Kolesnichenko, V.S. Marchenko,

and R.B. White

May 2004

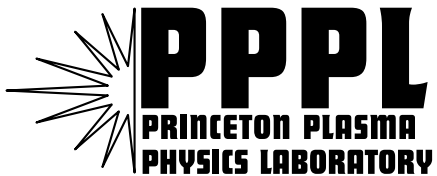

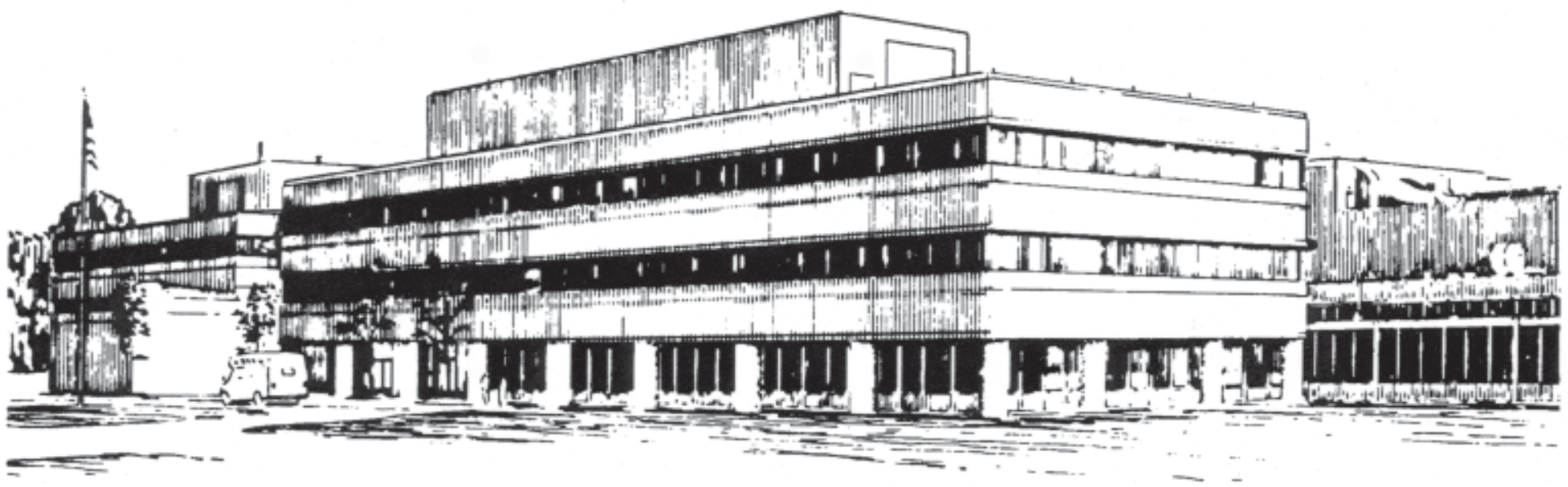

PRINCETON PLASMA PHYSICS LABORATORY PRINCETON UNIVERSITY, PRINCETON, NEW JERSEY 


\section{PPPL Reports Disclaimer}

This report was prepared as an account of work sponsored by an agency of the United States Government. Neither the United States Government nor any agency thereof, nor any of their employees, makes any warranty, express or implied, or assumes any legal liability or responsibility for the accuracy, completeness, or usefulness of any information, apparatus, product, or process disclosed, or represents that its use would not infringe privately owned rights. Reference herein to any specific commercial product, process, or service by trade name, trademark, manufacturer, or otherwise, does not necessarily constitute or imply its endorsement, recommendation, or favoring by the United States Government or any agency thereof. The views and opinions of authors expressed herein do not necessarily state or reflect those of the United States Government or any agency thereof.

\section{Availability}

This report is posted on the U.S. Department of Energy's Princeton Plasma Physics Laboratory Publications and Reports web site in Fiscal Year 2004. The home page for PPPL Reports and Publications is: http://www.pppl.gov/pub_report/

DOE and DOE Contractors can obtain copies of this report from:

U.S. Department of Energy

Office of Scientific and Technical Information

DOE Technical Information Services (DTIS)

P.O. Box 62

Oak Ridge, TN 37831

Telephone: (865) 576-8401

Fax: (865) 576-5728

Email: reports@adonis.osti.gov

This report is available to the general public from:

National Technical Information Service

U.S. Department of Commerce

5285 Port Royal Road

Springfield, VA 22161

Telephone: $1-800-553-6847$ or

(703) $605-6000$

Fax: (703) 321-8547

Internet: http://www.ntis.gov/ordering.htm 


\title{
Stabilization of sawtooth oscillations by the circulating energetic ions
}

\author{
Ya.I. Kolesnichenko*, V.S. Marchenko*, and R.B. White** \\ *Institute for Nuclear Research, Kyiv, 03680, Ukraine \\ ** Princeton Plasma Physics Laboratory, P.O. Box 451, Princeton, NJ, 08543, USA
}

(May 17, 2004)

\begin{abstract}
The influence of the well-circulating energetic ions on the ideal kink instability and semi-collisional tearing mode are studied. It is found that the precession of these ions can be a key factor that affects the instability: it can lead to the stabilization of the mentioned instabilities, the effect being weakly dependent on the direction of the injection. The developed theory is consistent with the experimental observations of the stabilization of sawtooth oscillations during the negative-ion-based neutral beam injection in JT-60U (G.J. Kramer et al., Nuclear Fusion 40, 1383 (2000)).
\end{abstract}


It is known that trapped energetic ions can stabilize sawtooth oscillations. This was shown in 1988 experimentally on JET, where the trapped energetic ions were produced during the Ion Cyclotron Resonance Heating (ICRH), ${ }^{1,2}$ and later theoretically. ${ }^{3,4}$ More recently it was found that the circulating ions stabilize sawteeth, too: a considerable extension of the sawtooth-free period has been observed in the JT-60 Upgrade tokamak during the Negativeion-based Neutral Beam Injection (NNBI). ${ }^{5,6}$ This stabilization cannot be attributed to the NNBI-produced change of the plasma pressure profile, $p(r)$ [the change of $p(r)$ affects the MHD potential energy ${ }^{7}$, because the change of the pressure was small. ${ }^{5}$ The current generated by NNBI also cannot explain the stabilization because the co-injection, which took place in the mentioned experiment on JT-60U, increases the magnetic shear at the $q=1$ surface [the increasing shear destabilizes (rather than stabilizes) the sawtooth instability ${ }^{8,9}$ ]. On the other hand, in the same shot there was no observable effect from the Positive-ionbased Neutral-Beam-Injection (PNBI), although the PNBI power was comparable to the NNBI power. This together with the fact that the particle energy during PNBI was much lower than during NNBI indicate that the nature of the observed stabilization is essentially kinetic (non-MHD) and can be produced only by particles with sufficiently high energy. This conclusion is in agreement with the kinetic theory of Ref. ${ }^{10}$ However, recently it was shown that the non-adiabatic contribution found in Ref. ${ }^{10}$ is exactly cancelled by the finite-orbit term in the fluid response. ${ }^{11}$ Instead, a new fluid term arising from the particles crossing the $q=1$ surface was obtained in $\operatorname{Ref}^{11}$. It was concluded in both mentioned works that the effect of the circulating ions is stabilizing only for the co-injection, the counter-injection was found to be destabilizing. In the present work, we develop a theory, which takes into account the average curvature drift of the circulating particles - the factor, which was ignored in previous theories.

Before to begin a stability analysis we note that the following resonance determines the interaction of the circulating particles and a perturbation:

$$
\omega-(m-n \bar{q}+S) \omega_{\theta}-n \omega_{d}=0
$$


where $\omega$ is the wave frequency, $\omega_{\theta}$ is the frequency of the of the particle motion in the poloidal direction, $\omega_{d}$ is the precession frequency defined by $\omega_{d}=\left\langle q v_{d}^{2}\right\rangle \equiv \oint q v_{d}^{2} d t / \tau_{b}$, $\mathbf{v}_{\mathbf{d}}$ is the particle drift velocity, the superscript denotes a contra-variant component in Boozer coordinates, $\tau_{b}$ is the particle transit time, $\bar{q}=\oint q d \theta /(2 \pi)$ with the integral taken along the particle orbit, $q$ is the safety factor, $m$ and $n$ are the poloidal mode number and toroidal mode number, respectively, $S$ is an integer. Equation (1) is written in the assumption that the perturbation has the form $\widetilde{X} \propto \exp (-i \omega t-i n \varphi+i m \vartheta)$. In the case of well-circulating particles and $|m-n \bar{q}| \ll|m|$, this resonance condition is reduced to

$$
\omega-\left(k_{\|}+\frac{S}{q R}\right) v_{\|}-n \omega_{d}=0,
$$

where $v_{\|} \approx$ const is the particle velocity along the magnetic field, $k_{\|}=(m-n q) / q R_{0}$, the bar over $q$ is omitted. In geometry with the shifted circular flux surfaces and large aspect ratio the precession frequency is given by ${ }^{12}$

$$
\begin{gathered}
\omega_{d}=\zeta q \frac{v_{\|}^{2}}{\Omega_{b} R_{0}^{2}}, \\
\zeta=\frac{3}{2}+s\left(\beta_{p}+\frac{l_{i}}{2}\right)+\frac{\alpha_{p}}{2 \epsilon q^{2}}-\frac{1}{q^{2}}-s \frac{\Delta_{b} R_{0}}{r^{2}}
\end{gathered}
$$

where $\Omega_{b}$ is the beam ion gyrofrequency, $s=r q^{\prime} / q$ is the magnetic shear, $l_{i}=$ $2 /\left(r^{2} B_{\theta}^{2}\right) \int_{0}^{r} B_{\theta}^{2} r d r$ is the internal inductance per unit length, $\beta_{p}=\left(8 \pi / B_{\theta}^{2}\right)(\bar{p}-p)$ with $\bar{p}=\left(2 / r^{2}\right) \int_{0}^{r} p r d r$ the average pressure, $\alpha_{p}=-\left(8 \pi p^{\prime} / B_{0}^{2}\right) R_{0} q^{2}, \Delta_{b}=q v_{\|} / \Omega_{b}$, and prime denotes the radial derivative. Note that the precessional frequency in Ref. ${ }^{12}$ is defined as $\omega_{D}=-\left\langle q v_{d}^{2}\right\rangle$, which explains why $\omega_{d}$ and $\omega_{D}$ have different signs.

The "toroidal circulation" resonance considered in Ref. ${ }^{10}$ corresponds to the case of $\omega_{d}=$ $0, S=1$. In contrast to this, we take into account that $\omega_{d} \neq 0$ but neglect the sideband resonances by putting $S=0$. Then the energetic particle functional, $\lambda_{k}$, can be easily obtained from that of Refs. ${ }^{13,14}$ where $\omega_{d}$ was neglected. We have: ${ }^{15}$

$$
\lambda_{k}=-\frac{\pi}{\left(B_{\theta} s\right)_{r_{s}}^{2}} \int_{0}^{r_{s}} r h(r) d r
$$




$$
h(r)=-\frac{8}{\pi} \frac{1}{R_{0}^{2}} \int d \vec{v} \varepsilon^{2} \frac{\partial F_{b}}{\partial P_{\phi}} \frac{\Delta_{b}}{\left|\Delta_{b}\right|} \int_{-\pi}^{\pi} \cos \theta \frac{H(1-|z|) \sqrt{1-z^{2}}}{\omega-k_{\|}\left(r_{s}+z \Delta_{b}\right) v_{\|}-\omega_{d}\left(r_{s}\right)} d \theta,
$$

where $z=\left[\bar{r}(r, \theta)-r_{s}\right] / \Delta_{b}, \bar{r}=r+\Delta_{b} \cos \theta, q\left(r_{s}\right)=1, \varepsilon$ is the particle energy, $P_{\phi}=$ $m_{b} v_{\|} R+e \psi / c$ is the canonical angular momentum with $m_{b}$ the particle mass and $\psi$ the poloidal flux, $H(x)$ is the Heavy-side step function. The factor $H(1-|z|)$ in Eq. (6) takes into account that only particles crossing the $q=1$ surface in the course of their drift motion considerably exchange the energy with the perturbations. For these particles the resonance condition determined by the denominator of Eq. (6) can be written as

$$
\omega+s_{1} \frac{v_{\|}^{2} z}{\Omega_{b} R_{0} r_{s}}-\frac{\zeta_{s} v_{\|}^{2}}{\Omega_{b} R_{0}^{2}}=0 .
$$

In order to calculate the integrals in Eq. (6) we have to specify the distribution function of the energetic ions. We take it in the form: ${ }^{13}$

$$
F_{b}=\frac{\sqrt{2} m_{b}^{3 / 2}}{\pi \varepsilon_{\alpha}} p_{b}(r) H\left(\varepsilon_{\alpha}-\varepsilon\right) \frac{\delta\left(\mu B_{0} / \varepsilon\right)}{\varepsilon_{c}^{3 / 2}+\varepsilon^{3 / 2}}\left[\frac{1}{2}(1-\sigma)+\sigma H\left( \pm v_{\|}\right)\right],
$$

where $p_{b}(r)$ is the beam ion pressure, $\varepsilon_{\alpha}$ is the injection energy, $\varepsilon_{c} \sim\left(m_{b} / m_{e}\right)^{1 / 3} T_{e}, T_{e}$ is the electron temperature, ( $\varepsilon_{c} \ll \varepsilon_{\alpha}$ for NNBI), $\sigma=0$ for the balanced injection, and $\sigma=1$ in the other cases, the signs "+" and "-" in the argument of the step function correspond to the co-injection and counter-injection, respectively. Combining Eqs. (5)-(8), we obtain:

$$
\lambda_{k}=\frac{2}{3} \frac{r_{s}}{\pi R_{0}}\left[\frac{\Delta_{b}^{\alpha}}{r_{p b}} \frac{\beta_{b \theta}}{s^{3}}\right]_{r_{s}} I(\Omega, \kappa)
$$

where

$$
\begin{gathered}
I(\Omega, \kappa) \equiv\left(1+3 \Omega^{2}+6 \Omega \kappa-\kappa^{2}\right) \ln \frac{\Omega+1-\kappa}{\Omega-1-\kappa}+10 \Omega-2 \kappa+ \\
4 \Omega^{3 / 2}\left[\frac{2 \kappa-1}{(\kappa-1)^{1 / 2}} \ln \frac{\sqrt{\Omega}+\sqrt{\kappa-1}}{\sqrt{\Omega}-\sqrt{\kappa-1}}-\frac{2 \kappa+1}{(\kappa+1)^{1 / 2}} \ln \frac{\sqrt{\Omega}+\sqrt{\kappa+1}}{\sqrt{\Omega}-\sqrt{\kappa+1}}\right],
\end{gathered}
$$

$\kappa \equiv \zeta_{s} \epsilon_{s} / s_{1}, \Omega=\omega / \omega_{s}$ with $\left.\omega_{s} \equiv k_{\|}\right|_{r_{s}-\Delta_{b}} v_{\|}=s v_{\| \alpha}^{2} /\left(\Omega_{b} R_{0} r_{s}\right), \beta_{b \theta}$ is the poloidal beta of beam ions, and $r_{p b}^{-1}=-d \ln p_{b} / d r$. Note that when $\kappa>1$ (this can be the case in high$\beta$ plasmas of spherical tori), Im $\lambda_{k} \rightarrow 0$ and, thus, the "diamagnetic" fishbone branch is stabilized. ${ }^{15}$ 
Below we assume that $|\Omega| \rightarrow 0$, which is relevant to the sawtooth instability. Then we obtain:

$$
\lambda_{k}(0)=\frac{2}{3} \frac{r_{s}}{\pi R_{0}}\left[\frac{\Delta_{b}^{\alpha}}{r_{p b}} \frac{\beta_{b \theta}}{s^{3}}\right]_{r_{s}}\left\{\left(1-\kappa^{2}\right) \ln \left|\frac{\kappa-1}{\kappa+1}\right|-2 \kappa-i \pi\left(1-\kappa^{2}\right) H(1-\kappa)\right\},
$$

where $\lambda_{k}(0)=\lambda_{k}(\Omega=0)$. It follows from Eq. (11) that $\operatorname{Re} \lambda_{k}(\Omega=0, \kappa=0)=0$, whereas the presence of the precession $(\kappa \neq 0)$ leads to $\operatorname{Re} \lambda_{k}(0)<0$. This means that the precession tends to stabilize the internal kink mode. The obtained stabilization by the precession is a consequence of the conservation of the magnetic flux linked through the energetic ion precessional drift orbits, as in the case of the trapped-ion stabilization. ${ }^{3,4}$ This non-MHD constraint becomes relevant when precession frequency considerably exceeds the ideal kink growth rate, i.e. when ${ }^{9}$

$$
\frac{\omega_{d}}{\omega_{A}} \gg \lambda_{c}
$$

where $\omega_{A} \equiv v_{A} /\left(\sqrt{3} R_{0}\right)$ is the Alfvén frequency, $\lambda_{c}$ is the negative of the normalized potential energy of the bulk plasma. ${ }^{7}$

Let us evaluate $\lambda_{k}$ in the JT-60U experiments, where the sawtooth stabilization by NNBI was observed (during the $\mathrm{D} \rightarrow \mathrm{H}$ co-injection), but there was no influence of the PNBI ions on sawteeth. ${ }^{5,6}$ We use the following parameters: $\varepsilon_{\alpha} \simeq 360 \mathrm{keV}, s_{1}=0.4, r_{s} \simeq 27 \mathrm{~cm}, R_{0} \simeq 3.2$ $\mathrm{m}, B_{0}=3.5 \mathrm{~T}, \beta_{p s} \simeq 0.5, l_{i} \simeq 1.41, \omega_{A} \simeq 8 \times 10^{6} \mathrm{~s}^{-1}$. Then we obtain from Eqs. (3), (4) that Eq. (12) is well satisfied for the NNBI ions $\left(\lambda_{c} \simeq 7 \times 10^{-4},{ }^{10} \omega_{d} / \omega_{A} \simeq 3 \times 10^{-3}\right)$. However, Eq. (12), which represents a necessary condition for the stabilization of sawteeth by the energetic ions, is difficult to satisfy for the PNBI ions $(\varepsilon<90 \mathrm{keV})$. Taking $\zeta_{s} \sim 1$ and $p_{b}(r)=p_{b 0}\left[1-(r / a)^{2}\right]^{6}$ with $a=82 \mathrm{~cm}, \beta_{b 0} \simeq 0.4 \%$, we obtain $\beta_{b \theta}\left(r_{s}\right) \simeq 0.29, r_{p b} \simeq 18$ $\mathrm{cm}, R_{0} / \Delta_{b}^{\alpha} \simeq 93, \kappa \simeq 0.3$. Equation (11) then yields $\operatorname{Re} \lambda_{k}(0) \simeq-1.8 \times 10^{-2}$. The absolute value of the calculated $\operatorname{Re} \lambda_{k}(0)$ well exceeds $\lambda_{c} \simeq 7 \times 10^{-4}$. We conclude from here that the ideal kink instability is suppressed by the NNBI in JT-60U.

However, when $\lambda_{c}+\lambda_{k}<0$, non-ideal instabilities (tearing mode) can arise. One can see that collisionless effects play an important role in this case: with the estimated magnetic 
Reynolds number $S_{M} \simeq 10^{7}$, the resistive width of the singular layer $\delta_{\eta} \equiv r_{s} /\left(s_{1} S_{M}\right)^{1 / 3} \simeq 1.7$ $\mathrm{mm}$ falls below the ion hybrid Larmor radius $\rho_{\tau} \equiv \rho_{i} \sqrt{1+T_{e} / T_{i}} \simeq 2.6 \mathrm{~mm}$ (we take $T_{i} \simeq T_{e}$ ), but exceeds the inertial skin depth, $d_{e} \equiv c / \omega_{p e} \simeq 1.2 \mathrm{~mm}$. Under these conditions and $\left|\omega / \omega_{A}\right|<\rho_{\tau} / r_{s}$ the dispersion relation can be written $\operatorname{as}^{16,17}$

$$
-\frac{\pi}{2}\left(\frac{\omega}{\omega_{A}}\right)^{2}=\frac{\rho_{\tau}}{r_{s}}\left(\lambda_{c}+\lambda_{k}(0)\right)+\left(\frac{i \omega_{A}}{\omega}\right)^{3 / 2}\left(\frac{\rho_{\tau}}{r_{s}}\right)^{2} S_{M}^{-1 / 2} s_{1}^{3}
$$

In the absence of fast ions we have an estimate $\lambda_{c} \ll(\pi / 2)^{3 / 7}\left(\rho_{\tau} / r_{s}\right)^{1 / 7} S_{M}^{-2 / 7} s_{1}^{12 / 7}$, and Eq. (13) describes the semi-collisional $m=1$ tearing mode ${ }^{17,9}$ with the growth rate $\gamma_{\rho} / \omega_{A}=$ $(2 / \pi)^{2 / 7}\left(\rho_{\tau} / r_{s}\right)^{4 / 7} S_{M}^{-1 / 7} s_{1}^{6 / 7}$. In the presence of fast ions we have $\lambda_{c}+\operatorname{Re} \lambda_{k}<0,\left|\lambda_{c}+\lambda_{k}(0)\right|>$ $\rho_{\tau} / r_{s} \gg(\pi / 2)^{3 / 7}\left(\rho_{\tau} / r_{s}\right)^{1 / 7} S_{M}^{-2 / 7} s_{1}^{12 / 7}$. A solution of Eq. (13) in this case is [cf. Eq. (21) of Ref. $\left.^{15}\right]:$

$$
\frac{\omega}{\omega_{A}}=i\left(\frac{\rho_{\tau}}{r_{s}}\right)^{2 / 3} s_{1}^{2} S_{M}^{-1 / 3}\left(\left|\lambda_{c}+\lambda_{k r}\right|+i\left|\lambda_{k i}\right|\right)^{-2 / 3}
$$

where $\lambda_{k r} \equiv \operatorname{Re} \lambda_{k}$ and $\lambda_{k i} \equiv \operatorname{Im} \lambda_{k}$. For the parameters given above Eq. (14) yields $\left|\omega_{* i} / \omega\right| \simeq$ 5, with $\left|\omega_{* i}\right| \simeq 10^{4} \mathrm{~s}^{-1}$ the bulk ion diamagnetic frequency. This means that the considered non-ideal branch is to be stabilized by the diamagnetic effects: according to Ref. ${ }^{9}$, the stabilization takes place when $\omega_{* i} / \gamma_{0} \gtrsim 2$, where $\gamma_{0}$ is the growth rate calculated for $\omega_{* i}=0$, i.e., the growth rate determined by Eq. (14). On the other hand, $|\omega| / \omega_{s} \simeq 0.02$, which is consistent with our assumption that $|\Omega| \rightarrow 0$.

Note that our estimates above were made in the assumption that the plasma crosssection is circular, while the plasma in the considered shot was moderately shaped ( with the elongation $\sim 1.46$ at the edge $\left.{ }^{6}\right)$. Nevertheless, because the condition $\left|\lambda_{k r}(0)\right| \gg \lambda_{c}$ is well satisfied, one can expect that it will be satisfied even with a moderately reduced precession of the energetic ions in shaped geometry.

Now we discuss how the obtained results are related to those in Ref. ${ }^{11}$. The total response of the beam ions crossing the $q=1$ surface is given by

$$
\operatorname{Re} \lambda_{t o t}=\frac{r_{s}}{3 R_{0}}\left(\frac{\Delta_{b}^{\alpha} \beta_{b \theta}}{r_{p b} s^{2}}\right)_{r=r_{s}}\left\{\frac{2}{\pi s_{1}}\left[\left(1-\kappa^{2}\right) \ln \frac{1-\kappa}{1+\kappa}-2 \kappa\right]+\sigma_{\lambda}\right\}
$$


where $\sigma_{\lambda}=(-1,0,1)$ for purely co-injection, balanced injection, and counter-injection, respectively. The second term in Eq. (15) is actually the fluid term found in Ref. ${ }^{11}$. It is less than the first term for the JT-60U parameters used above. In general, when $\kappa \ll 1$, the ratio of the first term to the second one is about $8 \zeta_{s} \epsilon_{s} /\left(\pi s_{1}^{2}\right)$, which exceeds unity for $\epsilon_{s} \sim s_{1}^{2}$ and $\zeta_{s} \sim 1$. In this case, Eq. (15) predicts the stability even for counter-injection. Note that the obtained results imply a stabilizing effect of the circulating $\alpha$-particles $\left(\sigma_{\lambda}=0\right)$ in fusion reactors.

In summary, we have shown that the circulating energetic ions can stabilize the ideal kink mode and semi-collisional tearing mode. The stabilization results from the finite magnitude of the particle precession, which affects the resonance interaction of the perturbations and the particles. The developed theory predicts the stabilization independently on the direction of the circulation and for the balanced injection, although the found stabilizing effect depends on the direction of the circulation [see Eqs. (4), (15)]. As the precession of the circulating particles is rather sensitive to many factors (the plasma shaping, the magnetic shear, the profile of the plasma pressure, etc. ${ }^{12}$ ), the considered stabilizing mechanism is not so robust as the corresponding mechanism associated with the trapped particles. The developed theory seems to explain the stabilization of the sawtooth instability observed in the experiments with NNBI on JT-60U: the made estimates show that the new mechanism dominates over the mechanism suggested in Ref. ${ }^{11}$, although the calculation of the precession frequency with taking into both account the plasma shaping and the magnetic shear is required for the final conclusion. In addition, the developed theory is consistent with the fact that the PNBI had no influence on the sawtooth oscillations in JT-60U.

\section{ACKNOWLEDGMENTS}

The research described in this publication was made possible in part by the Award No. UP2-2419-KV-02 of the U.S. Civilian Research \& Development Foundation and the Government of Ukraine and the U.S. Department of Energy under contract number DE- 
AC02-76-CHO3073. 


\section{REFERENCES}

${ }^{1}$ D.J. Campbell et al., Phys. Rev. Lett. 60, 2148 (1988).

2 D.J. Campbell et al., Proc. 15th EPS Conf. on Contr. Fusion and Plasma Heating, v. 12B, Part 1, p.377 (1988).

${ }^{3}$ R.B. White, F. Romanelli, M.N. Bussac, Phys. Fluids B4, 795 (1990).

${ }^{4}$ F. Porcelli, Plasma Phys. Control. Fusion 33, 1601 (1991).

${ }^{5}$ K. Tobita, K. Hammatsu, M. Iwase, T. Suzuki, Y. Koide, S. Moriyama, T. Nishitani, Y. Kusama, M. Nemoto, and A. Morioka, in Proceedings of the 6th IAEA Technical Committee Meeting on Energetic Particles in Magnetic Confinement Systems, 1999, JAERI, Naka, Japan (Japan Atomic Energy Research Institute, Tokai-mura, Nak-gun, Ibaraki-ken, Japan, 2000), p.73.

${ }^{6}$ G.J. Kramer, M. Iwase, Y. Kusama, A. Morioka, M. Nemoto, T. Nichitani, K. Shinohara, S. Takeji, K. Tobita, T. Ozeki, C.Z. Cheng, G.-Y. Fu, and R. Nazikian, Nucl. Fusion 40, $1383(2000)$.

${ }^{7}$ M.N. Bussac, R. Pellat, D. Edery, and J.L. Soule, Phys. Rev. Lett. 35, 1638 (1975).

${ }^{8}$ F.M. Levinton, et al., Phys. Rev. Lett. 72, 2895 (1994).

${ }^{9}$ F. Porcelli, D. Boucher, and M.N. Rosenbluth, Plasma Phys. Control. Fusion 38, 2163 (1996).

${ }^{10}$ S. Wang, T. Ozeki, and K. Tobita, Phys. Rev. Lett. 88, 105004 (2002).

11 J.P. Graves, Phys. Rev. Letters 92 185003-1 (2004).

${ }^{12}$ Ya.I. Kolesnichenko, R.B. White, and Yu.V. Yakovenko, Phys. Plasmas 10, 1449 (2003).

${ }^{13}$ R. Betti and J.P. Freidberg, Phys. Rev. Lett. 70, 3428 (1993).

${ }^{14}$ R. Betti, Plasma Phys. Control. Fusion 35, 941 (1993). 
${ }^{15}$ Ya.I. Kolesnichenko, V.S. Marchenko, and R.B. White, Phys. Plasmas 8, 3143 (2001).

${ }^{16}$ F. Porcelli, Phys. Rev. Lett. 66, 425 (1991).

${ }^{17}$ F. Pegoraro, F. Porcelli, and T.J. Schep, Phys. Fluids B1, 364 (1989). 


\section{External Distribution}

Plasma Research Laboratory, Australian National University, Australia

Professor I.R. Jones, Flinders University, Australia

Professor João Canalle, Instituto de Fisica DEQ/IF - UERJ, Brazil

Mr. Gerson O. Ludwig, Instituto Nacional de Pesquisas, Brazil

Dr. P.H. Sakanaka, Instituto Fisica, Brazil

The Librarian, Culham Laboratory, England

Mrs. S.A. Hutchinson, JET Library, England

Professor M.N. Bussac, Ecole Polytechnique, France

Librarian, Max-Planck-Institut für Plasmaphysik, Germany

Jolan Moldvai, Reports Library, Hungarian Academy of Sciences, Central Research Institute for Physics, Hungary

Dr. P. Kaw, Institute for Plasma Research, India

Ms. P.J. Pathak, Librarian, Institute for Plasma Research, India

Ms. Clelia De Palo, Associazione EURATOM-ENEA, Italy

Dr. G. Grosso, Instituto di Fisica del Plasma, Italy

Librarian, Naka Fusion Research Establishment, JAERI, Japan

Library, Laboratory for Complex Energy Processes, Institute for Advanced Study, Kyoto University, Japan

Research Information Center, National Institute for Fusion Science, Japan

Dr. O. Mitarai, Kyushu Tokai University, Japan

Dr. Jiangang Li, Institute of Plasma Physics, Chinese Academy of Sciences, People's Republic of China

Professor Yuping Huo, School of Physical Science and Technology, People's Republic of China

Library, Academia Sinica, Institute of Plasma Physics, People's Republic of China

Librarian, Institute of Physics, Chinese Academy of Sciences, People's Republic of China

Dr. S. Mirnov, TRINITI, Troitsk, Russian Federation, Russia

Dr. V.S. Strelkov, Kurchatov Institute, Russian Federation, Russia

Professor Peter Lukac, Katedra Fyziky Plazmy MFF UK, Mlynska dolina F-2, Komenskeho Univerzita, SK-842 15 Bratislava, Slovakia

Dr. G.S. Lee, Korea Basic Science Institute, South Korea

Institute for Plasma Research, University of Maryland, USA

Librarian, Fusion Energy Division, Oak Ridge National Laboratory, USA

Librarian, Institute of Fusion Studies, University of Texas, USA

Librarian, Magnetic Fusion Program, Lawrence Livermore National Laboratory, USA

Library, General Atomics, USA

Plasma Physics Group, Fusion Energy Research Program, University of California at San Diego, USA

Plasma Physics Library, Columbia University, USA

Alkesh Punjabi, Center for Fusion Research and Training, Hampton University, USA

Dr. W.M. Stacey, Fusion Research Center, Georgia Institute of Technology, USA

Dr. John Willis, U.S. Department of Energy, Office of Fusion Energy Sciences, USA

Mr. Paul H. Wright, Indianapolis, Indiana, USA 
The Princeton Plasma Physics Laboratory is operated by Princeton University under contract with the U.S. Department of Energy.

\author{
Information Services \\ Princeton Plasma Physics Laboratory \\ P.O. Box 451 \\ Princeton, NJ 08543
}

Phone: 609-243-2750

Fax: 609-243-2751

e-mail: pppl_info@pppl.gov

Internet Address: http://www.pppl.gov 